\title{
Electromagnetic metamaterial with built-in microstructures
}

\author{
Jensen $\mathrm{Li}$ and C. T. Chan \\ Department of Physics, The Hong Kong University of Science and Technology, Clear Water Bay, Kowloon, Hong Kong, China
}

(Received 22 June 2005; revised manuscript received 12 September 2005; published 14 November 2005)

\begin{abstract}
Electromagnetic metamaterial, which is usually treated as a homogeneous medium, can manipulate both the far fields and the near fields. On the other hand, due to its artificially structured nature, near fields of high spatial frequencies invoke the nonlocal response of the medium. Here, we study this nonlocality using a dipolar model. From the model, we can derive the effective medium directly and the complete $(k, \omega)$ dispersion is used as the starting point in describing the material. We apply the model on the superlensing effect via a slab of double-negative metamaterial. In a particular case the wavelength is a hundred times of the lattice spacing, subwavelength imaging is sensible only when the lens is thinner than three wavelengths. The resolution can be improved by employing a smaller lattice spacing.
\end{abstract}

DOI: $10.1103 /$ PhysRevB.72.195103

PACS number(s): 78.20.Ci, 42.30.Wb, 73.20.Mf, 42.70.Qs

\section{INTRODUCTION}

A double-negative (DNG) medium for electromagnetic (EM) waves, with simultaneously negative permittivity and negative permeability, was originally conceived by Veselago ${ }^{1}$ as an isotropic and homogeneous local medium. These materials are also called metamaterials as they are not found in nature, and must be made artificially. ${ }^{2}$ Amazing properties of DNG media, such as the perfect lens imaging ${ }^{3}$ that happens at $\varepsilon=\mu=-1$, is also deduced in a homogeneous medium treatment. The lens gives a perfect image but at the same time, there is an inevitable divergence of fields on the exit interface. $^{4,5}$ Previous works circumvented the problem by adding a small amount of absorption or introducing some mismatch in the impedance. The lens is then no longer perfect but imaging with subwavelength resolution can still be achieved. ${ }^{6-14}$ Alternatively, the divergence can be avoided by considering the time evolution of the image formation instead of the stationary state. ${ }^{15}$ In this case, a perfect resolution requires an infinite amount of time to develop. However, both approaches are not entirely satisfactory since the divergence stems from the use of the local effective medium description of the DNG medium near the perfect lens condition.

All realizations of DNG media to date are through resonance structures. The validity of a local effective medium hinges on the fact (or assumption) that the wavelength under consideration is much larger than the structural length scale so that it is meaningful to talk about an effective $\varepsilon$ and an effective $\mu$; and that the effective medium provides a good description. However, a lens having a resolution close to the lattice spacing must utilize the near fields of high spatial frequencies. It implies that the microscopic details of DNG media become important. Therefore, a model incorporating the microscopic structure is in principle needed for a proper discussion of the image resolution. We note that the need of extending the local effective medium in describing metamaterials is proposed ${ }^{16}$ without a quantitative model and recently, the Lindhard nonlocal permittivity for Fermi liquid of electrons is used for considering the near-field focusing by a silver slab. ${ }^{17,18}$ We will cast the discussion within a welldefined and concrete model and we will examine quantita- tively the difference between a truly homogeneous doublenegative slab and that has an underlying microstructure giving rise to effective double negativity. Here, the local effective medium is extended for including high spatial frequencies. The emphasis of this paper is not on the applicability of effective medium theories when $\varepsilon_{\text {eff }}$ or $\mu_{\text {eff }}$ tends to infinity or the extraction of $\varepsilon_{\text {eff }}$ and $\mu_{\text {eff }}$ from an artificial structure $^{19}$ but to explore the similarities and/or differences between a microstructured medium and its corresponding homogenized counterpart. The lattice spacing here is very small compared to the wavelength.

In conventional optics with the source-to-image distance $(l)$ much larger than the wavelength $(\lambda)$, the image resolution is always limited by $\lambda$. On the other hand, the resolution of the perfect lens in its homogeneous medium version is unlimited. The presence of the microstructure is expected to limit its resolution by a value in the order of the lattice spacing. ${ }^{20}$ However, we shall see that with the effect of spatial dispersion (due to the presence of the microstructure), the classical diffraction limit is recovered when $l \gg \lambda$. In other words, the lens acts as a super lens in most cases but it behaves as a conventional lens when $l \gg \lambda$.

This paper is organized as follows. In Sec. II, we will first establish a microscopic model of DNG medium in which the resonances are derived from an ordered array of dipoles of given polarizabilities. Once the polarizabilities of the dipoles are specified, the complete band structure can be determined. It is used as the basic starting point to describe the DNG medium. In Sec. III, the validity of the effective medium will be discussed through an example. After confirming the usefulness of the model, we will examine the superlensing effect of a DNG slab (refractive index $n=-1$ and intrinsic impedance $\eta \neq 1$ ) with impedance not matched within the context of the new model. In Sec. IV, we will go over to the perfect lens $(n=-1, \eta=1)$. We will examine the lens when $l \gg \lambda$ and Sec. V gives a summary of the paper.

\section{THE DIPOLAR MODEL}

The negative refractive index originates from the negative response of the individual resonating units embedded in the 
medium. Without loss of generality, let us use a spherical surface to enclose each individual resonating unit completely. The wave outside the spherical surface can be spanned by a multipole expansion. We treat each individual unit as a black box, and replace its effect on external EM waves by an electric polarizability tensor and a magnetic polarizability tensor, and also the response functions of higher multipoles. This approach mimics the derivation from the microscopic to the macroscopic Maxwell equations (see Sec. 6.6 in Ref. 21). The most popular realization of the metamaterials currently employs the metallic split rings and the metallic rods as the resonating units. These individual resonating units serve to make an in-phase magnetic dipole (split rings) to the magnetic field and an out-of-phase electric dipole (finite length metallic rods) to the electric field. Therefore, it is reasonable to neglect, at least for mathematical convenience, the higher multipoles in order to simplify the discussion of the physical phenomena displayed by the negative refraction. If the resonating units have a small number density, this model can even give quantitatively accurate results.

Let us put the resonators in a periodic lattice. Suppose there is a Bloch state of wave vector $\vec{q}$ (within the first Brillouin zone) in the lattice of the same type of resonating units of the metamaterial, the electric and the magnetic dipole moments at each lattice site $\vec{R}$ are given by

$$
\vec{p}(\vec{R})=\vec{p}_{0} e^{i \vec{q} \cdot \vec{R}}, \quad \vec{m}(\vec{R})=\vec{m}_{0} e^{i \vec{q} \cdot \vec{R}} .
$$

The local field at the origin site is thus expressed by

$$
\begin{aligned}
& \varepsilon_{0} \vec{E}_{\text {local }}=\sum_{|\vec{R}|>0} e^{i \vec{q} \cdot \vec{R}}\left(q_{0}^{2} \overleftrightarrow{G}(\vec{R}) \cdot \vec{p}_{0}-\frac{1}{c}\left(i q_{0} \nabla \times \overleftrightarrow{G}\right)(\vec{R}) \cdot \vec{m}_{0}\right), \\
& \frac{1}{\mu_{0}} \vec{B}_{\text {local }}=\sum_{|\vec{R}|>0} e^{i \vec{q} \cdot \vec{R}}\left(q_{0}^{2} \stackrel{\leftrightarrow}{G}(\vec{R}) \cdot \vec{m}_{0}+c\left(i q_{0} \nabla \times \overleftrightarrow{G}\right)(\vec{R}) \cdot \vec{p}_{0}\right),
\end{aligned}
$$

while the resonating units response to the local field through the electric polarizability, $\alpha_{e}$, and the magnetic polarizability, $\alpha_{m}$, of a single unit by

$$
\begin{aligned}
& \vec{p}_{0}=\varepsilon_{0} \alpha_{e}(\omega) \vec{E}_{\text {local }}, \\
& \vec{m}_{0}=\frac{1}{\mu_{0}} \alpha_{m}(\omega) \vec{B}_{\text {local }} .
\end{aligned}
$$

Here, $q_{0}$ is the wave number of the background (vacuum) and it is related to the speed of light, the permittivity and the permeability of vacuum by $q_{0}=\omega / c=\omega \sqrt{\varepsilon_{0} \mu_{0}}$. The Green's tensor for the background medium is given by

$$
\overleftrightarrow{G}(\vec{r})=\left(\overleftrightarrow{I}+\frac{1}{q_{0}^{2}} \nabla \nabla\right) \frac{e^{i q_{0} r}}{4 \pi r} .
$$

The dispersion relationship for the lattice can be solved by combining Eqs. (2) and (3). It is the multiple-scattering formulation ${ }^{22}$ up to the dipolar term. At the point, the formulation neither requires us to work at a low frequency nor must the dipoles vary slowly from site to site. However, for metamaterials which can be treated as a homogeneous medium in the lowest order approximation, it is usually reasonable to confine ourselves to the regime of the local effective medium approximation (low frequency limit with the reciprocal wave vector approaches the $\Gamma$ point) stated by

$$
q_{0} a \ll 1 \quad \text { and }\left|q_{i} a\right| \ll 1 \text { with } i=x, y, z,
$$

where $a$ is the lattice constant. It is more convenient to express the local field at the origin site based on the Lorentzcavity approach. Equation (2) is now rewritten as

$$
\begin{gathered}
\varepsilon_{0} \vec{E}_{\text {local }}=\varepsilon_{0} \vec{E}_{0}+\frac{m}{a^{3}} \mathrm{~F}\left[q_{0}^{2} \stackrel{\leftrightarrow}{G} \cdot \vec{p}_{0}-\frac{1}{c} i q_{0} \nabla \times \overleftrightarrow{G} \cdot \vec{m}_{0}\right], \\
\frac{1}{\mu_{0}} \vec{B}_{\text {local }}=\frac{1}{\mu_{0}} \vec{B}_{0}+\frac{m}{a^{3}}\left(\mathrm{~F}\left[q_{0}^{2} \overleftrightarrow{G} \cdot \vec{m}_{0}+c i q_{0} \nabla \times \overleftrightarrow{G} \cdot \vec{p}_{0}\right]-\vec{m}_{0}\right),
\end{gathered}
$$

where the transform Im is defined by

$$
\operatorname{Im}[f(\vec{r})](\vec{q})=\lim _{R_{0} \rightarrow \infty} \frac{a^{3}}{m} \sum_{0<|\vec{R}|<R_{0}} f(\vec{R}) e^{i \vec{q} \cdot \vec{R}}-\int_{0}^{R_{0}} d r^{3} f(\vec{r}) e^{i \vec{q} \cdot \vec{r}},
$$

$\mathrm{a}^{3} / \mathrm{m}$ is the volume occupied by a single unit, and $m$ has the values 1, 2, 4 for $\mathrm{SC}, \mathrm{BCC}, \mathrm{FCC}$, respectively, if we are considering cubic lattice structures. Note that the discrete lattice sum excludes the origin site but the continuum integral includes the origin.

$\vec{E}_{0}$ in the first term of Eq. (6) is related to the macroscopic field by

$$
\begin{aligned}
& \vec{E}(\vec{r})=\vec{E}_{0} e^{i \vec{q} \cdot \vec{r}}, \\
& \vec{B}(\vec{r})=\vec{B}_{0} e^{i \vec{q} \cdot \vec{r}},
\end{aligned}
$$

and the macroscopic $\vec{P}(\vec{r})$ and $\vec{M}(\vec{r})$ are related to $\vec{p}_{0}$ and $\vec{m}_{0}$ by

$$
\begin{aligned}
\vec{P}(\vec{r}) & =\frac{m}{a^{3}} \vec{p}_{0} e^{i \vec{q} \cdot \vec{r}}, \\
\vec{M}(\vec{r}) & =\frac{m}{a^{3}} \vec{m}_{0} e^{i \vec{q} \cdot \vec{r}} .
\end{aligned}
$$

These macroscopic quantities satisfy the macroscopic Maxwell equations (with time dependence factor $e^{-i \omega t}$ and S.I. units)

$$
\begin{gathered}
\nabla \times(\nabla \times \vec{E})-q_{0}^{2} \vec{E}=i \omega \mu_{0}(\nabla \times \vec{M}-i \omega \vec{P}), \\
\nabla \times(\nabla \times \vec{B})-q_{0}^{2} \vec{B}=\mu_{0} \nabla \times(\nabla \times \vec{M}-i \omega \vec{P}),
\end{gathered}
$$

which govern the propagation of the macroscopic fields generated by the sources. The solution of Eq. (10) is thus given by 


$$
\begin{aligned}
& \varepsilon_{0} \vec{E}=\left(\frac{q_{0}^{2}}{q^{2}-q_{0}^{2}}(\stackrel{\leftrightarrow}{I}-\hat{q} \hat{q})-\hat{q} \hat{q}\right) \cdot \vec{P}-\frac{1}{c} \frac{q_{0}}{q^{2}-q_{0}^{2}} \vec{q} \times \vec{M}, \\
& \frac{1}{\mu_{0}} \vec{B}=\left(\frac{q_{0}^{2}}{q^{2}-q_{0}^{2}}(\stackrel{I}{I}-\hat{q} \hat{q})-\hat{q} \hat{q}\right) \cdot \vec{M}+c \frac{q_{0}}{q^{2}-q_{0}^{2}} \vec{q} \times \vec{P},
\end{aligned}
$$

where the terms with denominator $q^{2}-q_{0}^{2}$ represent the interaction between the Bloch state and the light line. We note that when the Bloch wave vector of the sources $(\vec{q})$ matches any wave vectors of the medium (vacuum), resonance will be excited.

The second term in Eq. (6) denotes the correction of the near field due to the difference between discrete and continuum dipoles. In the low frequency regime $\left(q_{0} a \ll 1\right)$, there exists a finite value for $R_{0}$, the radius of the Lorentz sphere, to make the limit in Eq. (7) convergent. Outside the Lorentz sphere, the discrete dipoles can be treated as a continuum with respect to the central site at origin. The dipoles inside the Lorentz sphere mainly emit the quasistatic field to the central site. Therefore, it is a good approximation to expand the second term in Eq. (6) in a Taylor series of $q_{0}$. After some straightforward algebra with the assumption that the reciprocal wave vector $\vec{q}$ approaches the Brillouin zone center $(\Gamma$ point), the local fields can be further simplified to become

$$
\begin{aligned}
& \varepsilon_{0} \vec{E}_{\text {local }}=\varepsilon_{0} \vec{E}_{0}+\frac{1}{3} \frac{\vec{p}_{0}}{a^{3} / m}+q_{0}^{2} \mathrm{~F}\left[\frac{\stackrel{\leftrightarrow}{I}+\hat{r} \hat{r}}{8 \pi r}\right](\overrightarrow{0}) \frac{\vec{p}_{0}}{a^{3} / m}-i q_{0}^{3} \frac{\vec{p}_{0}}{6 \pi}, \\
& \frac{1}{\mu_{0}} \vec{B}_{\text {local }}=\frac{1}{\mu_{0}} \vec{B}_{0}-\frac{2}{3} \frac{\vec{m}_{0}}{a^{3} / m}+q_{0}^{2} \mathrm{~F}\left[\frac{\stackrel{\leftrightarrow}{I} \hat{r} \hat{r}}{8 \pi r}\right](\overrightarrow{0}) \frac{\vec{m}_{0}}{a^{3} / m}-i q_{0}^{3} \frac{\vec{m}_{0}}{6 \pi} .
\end{aligned}
$$

Equation (12) is the correct formula for the dynamic local field in the local effective medium approximation up to the third order of frequency. And for simplicity, we confine ourselves to one of the cubic lattices (SC, FCC, BCC) such that

$$
\mathrm{F}\left[\frac{\stackrel{\leftrightarrow}{I} \hat{r} \hat{r}}{8 \pi r}\right](\overrightarrow{0})=\mathrm{F}\left[\frac{1}{6 \pi r}\right](\overrightarrow{0}) \stackrel{\leftrightarrow}{I}=-\gamma\left(\frac{a}{2 \pi}\right)^{2} .
$$

For example, $\gamma=2.4$ for FCC, 5.942 for SC. By combining Eq. (12) and Eq. (3), we obtain

$$
\begin{gathered}
\vec{P}=\varepsilon_{0}(\varepsilon-1) \vec{E}, \\
\vec{M}=\frac{1}{\mu_{0}}\left(1-\frac{1}{\mu}\right) \vec{B},
\end{gathered}
$$

where the local effective permittivity $\varepsilon$ and the local effective permeability $\mu$ are defined relative to vacuum and they are given by the dynamic Clausius-Mossotti (CM) equations,

$$
\begin{aligned}
& \frac{\varepsilon+2}{\varepsilon-1}-3 \gamma\left(\frac{q_{0} a}{2 \pi}\right)^{2}=3 \frac{a^{3}}{m}\left(\frac{1}{\alpha_{e}(\omega)}+\frac{i q_{0}^{3}}{6 \pi}\right), \\
& \frac{\mu+2}{\mu-1}-3 \gamma\left(\frac{q_{0} a}{2 \pi}\right)^{2}=3 \frac{a^{3}}{m}\left(\frac{1}{\alpha_{m}(\omega)}+\frac{i q_{0}^{3}}{6 \pi}\right) .
\end{aligned}
$$

The first and the second terms in Eq. (12) are the familiar quasistatic contributions to the local field. The $q_{0}^{2}$ term is the dynamic correction due to the lattice structure. The $q_{0}^{3}$ term is the radiative-correction term such that the right-hand side of Eq. (15) remains purely real for nonabsorbing unit giving purely real $\varepsilon$ and purely real $\mu$. The dispersion near the $\Gamma$ point can be thus obtained from solving the macroscopic Maxwell equations [Eq. (10)] with the constitutive relationship [Eq. (14)], i.e., $q^{2}=q_{0}^{2} \varepsilon \mu$. These equations state that once a geometric structure is specified, there is a unique relationship between the microscopic polarizabilities and the macroscopic effective constitutive parameters, and we note that these equations are not "assumed," but have been derived explicitly. The above analysis is true for any Bloch wave of $q a \ll 1$. It also holds for an arbitrary slow varying distribution of dipoles (or slow varying macroscopic fields) deep inside the sample.

On the other hand, when the reciprocal wave vector is far away from the light line (near the Brillouin zone edge), the interaction terms in Eq. (11) become unimportant. Therefore, the coupling between the electric and the magnetic dipoles is minimal and the dispersion can be approximately decoupled into two separate eigenvalue problems,

$$
\begin{gathered}
\sum_{|\vec{R}|>0} q_{0}^{2} \stackrel{\leftrightarrow}{G}(\vec{R}) e^{i \vec{q} \cdot \vec{R}} \cdot \vec{p}_{0}=\frac{1}{\alpha_{e}(\omega)} \vec{p}_{0}, \\
\sum_{|\vec{R}|>0} q_{0}^{2} \stackrel{\leftrightarrow}{G}(\vec{R}) e^{i \vec{q} \cdot \vec{R}} \cdot \vec{m}_{0}=\frac{1}{\alpha_{m}(\omega)} \vec{m}_{0} .
\end{gathered}
$$

\section{EXTENSION FROM LOCAL EFFECTIVE MEDIUM DESCRIPTION OF DNG MEDIUM}

\section{A. Bulk properties}

In the preceding section, the local effective medium holds for $\vec{q}$ near the $\Gamma$ point. For $\vec{q}$ far away from the $\Gamma$ point, the full band structure must be considered. As an example, suppose that a DNG medium possesses $\varepsilon$ and $\mu$ in the form given in the literature (typically determined from an experiment measuring the transmittance and reflectance for an incident propagating wave $^{23}$ ),

$$
\begin{gathered}
\varepsilon(f)=1+\frac{25}{0.9^{2}-f^{2}}, \\
\mu(f)=1+\frac{9}{0.902^{2}-f^{2}},
\end{gathered}
$$

where $f$ is the frequency in GHz. From Eq. (15), we obtain the single particle polarizabilities $\alpha_{e}$ and $\alpha_{m}$ that give rise to such $\varepsilon$ and $\mu$. They are plotted in Fig. 1. We see that the electric resonance gives rise to a negative $\varepsilon$ while the magnetic resonance gives rise to a negative $\mu$.

On the other hand, the DNG medium is modeled as a lattice of dipolar units, and the local effective medium can be extended. The resonators are assumed to occupy an FCC structure of lattice constant $a=3 \mathrm{~mm}$. Figure 2 compares the 


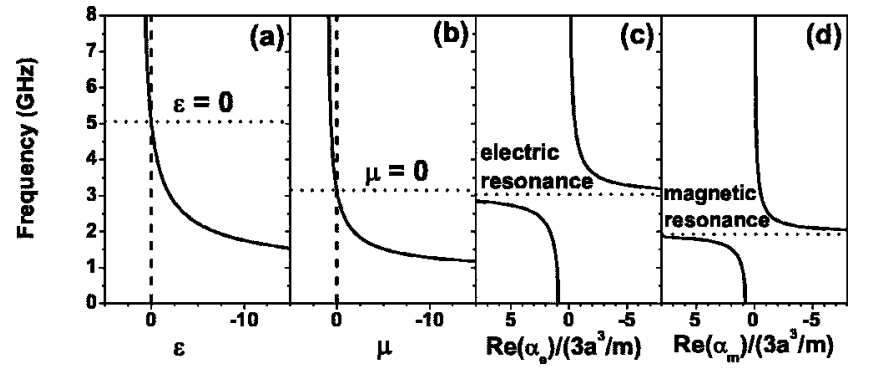

FIG. 1. Effective medium of (a) permittivity and (b) permeability given by Eq. (17) and the corresponding single-particle (c) electric polarizability and (d) magnetic polarizability in the dipolar model. See Eq. (15) for their relationship.

full band structures for either one polarizability "turned on" (the other one is set to zero) or both of them turned on simultaneously. The left-hand panels show the results based on the dipolar model [multiple-scattering formulation, solving Eq. (2) with Eq. (3)]. The right-hand panels show the corresponding dispersion in the local effective medium treatment.

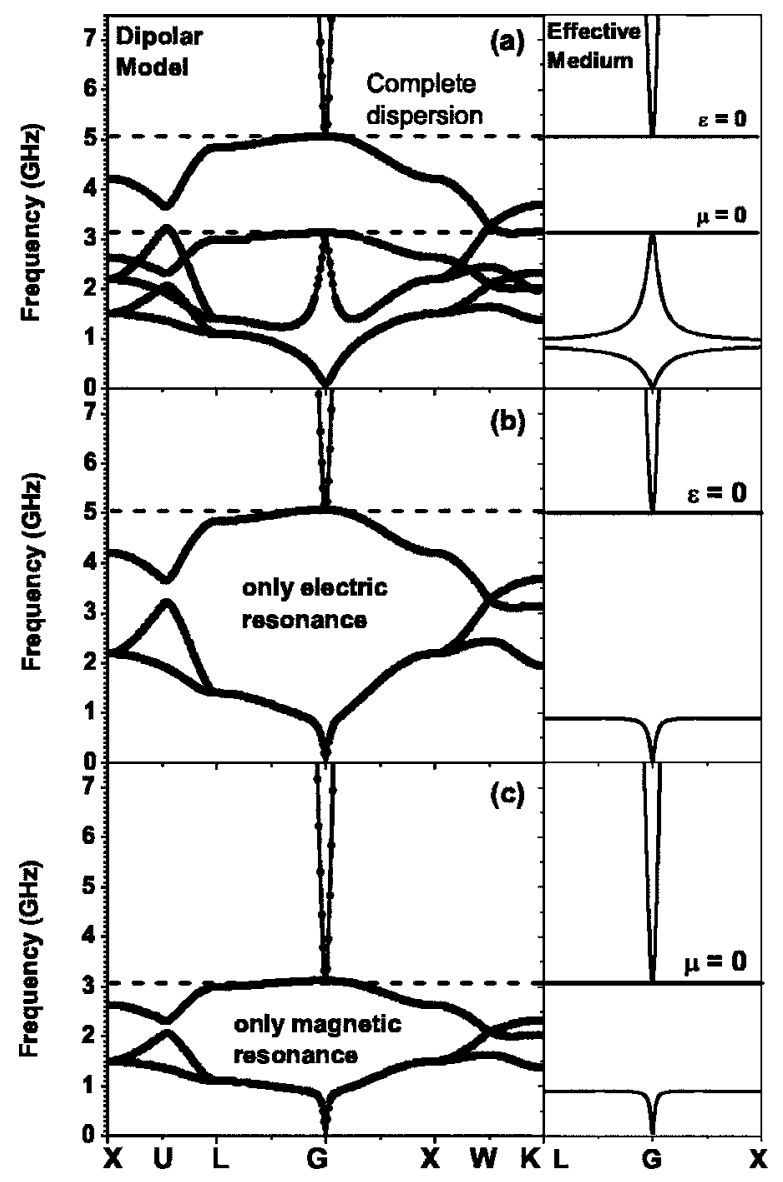

FIG. 2. Full band structure of the effective medium given by Eq. (17) with microstructure (FCC structure with lattice constant of 3 $\mathrm{mm}$ ) when (a) both electric and magnetic polarizabilities are turned on; (b) only electric polarizability $\alpha_{e}$ is turned on; (c) only magnetic polarizability $\alpha_{m}$ is turned on. The right-hand panels show the corresponding dispersion diagrams in the local effective medium description.
Let us first concentrate on the case where we only turn on $\alpha_{e}$ with $\alpha_{m}=0 .{ }^{24}$ The electric resonance opens a band gap at the resonating frequency. Near the $\Gamma$ point, the doubly degenerate transverse bands ( the linear band starting from zero frequency and the parabolic band starting from $5.1 \mathrm{GHz}$, governed by $q^{2}=q_{0}^{2} \varepsilon$ with $\vec{q} \cdot \vec{E}=\vec{q} \cdot \vec{H}=0$ ) and the relatively flat longitudinal band ( $\varepsilon=0$ with $\vec{q} \| \vec{E}$, meeting with a transverse band at the $\Gamma$ point) are well approximated by the effective medium. A similar physical picture holds for the case when only $\alpha_{m}$ is turned on, shown in Fig. 2(c). When both $\alpha_{e}$ and $\alpha_{m}$ are turned on, shown in Fig. 2(a), an extra band appears in the frequency range where the two gaps arising from the two resonances overlap. It is denoted as the negative refractive band. Near the $\Gamma$ point, it is doubly degenerate. It has two transverse modes satisfying $q^{2}=q_{0}^{2} \varepsilon \mu$ with both negative $\varepsilon$ and negative $\mu$. This is an explicit demonstration that by combining microscopic resonators which individually give rise to negative $\varepsilon$ and negative $\mu$, a propagating band of negative group velocity appears near the zone center in the frequency range that was originally forbidden for either the electric or the magnetic resonance.

In all three cases, the local effective medium provides a nearly exact description near the $\Gamma$ point, but it breaks down when we move far away from it. This happens at frequencies in which $\varepsilon$ or $\mu$ diverges. This breakdown explains why the longitudinal band is not exactly flat and eigenmodes exist even in the frequency regime where $\varepsilon$ and $\mu$ have opposite signs ( $0.9 \mathrm{GHz}$ upward to the longitudinal band). When $\vec{q}$ is far away from the $\Gamma$ point, the dispersion decouples [see Eq. (16)]. The bands should be labeled as either magnetic or electric instead. For example, an electric mode is specified by $\vec{P}$ alone with $\vec{M}=0$. Its polarization is not perpendicular or parallel to $\vec{q}$ in general. The decoupling in this region of Brillouin zone means that the three electric bands [from Fig. 2(b)] and the three magnetic bands [from Fig. 2(c)] stay at the same positions when both resonances are turned on, as shown in Fig. 2(a).

In short, the complete dispersion in the low frequency regime is obtained from overlapping the electric $\left(\alpha_{m}=0\right)$ and the magnetic bands $\left(\alpha_{e}=0\right)$ for $\vec{q}$ far away from the $\Gamma$ point and from the effective medium $\left(q^{2}=q_{0}^{2} \varepsilon \mu\right)$ for $\vec{q}$ near the $\Gamma$ point. Next, we investigate how well the local effective medium represents the true composite medium with microstructures in its optical properties. We pay particular attention to the focusing effect of the DNG slab by considering the far fields (propagating waves) and then the near fields (evanescent waves) emitting from a point source.

\section{B. Slab properties, far field}

The dipolar model and the local effective medium are tested with the case that we have propagating input waves on a DNG slab.

The shaded area in Fig. 3(a) shows the (111)-projected band structure of the same configuration in Fig. 2(a). The two parabolic red solid lines mark the boundary of the bulk states of the medium by $\left|\vec{q}_{t}\right| \leqslant q_{0}|\sqrt{\varepsilon} \sqrt{\mu}|$ where $\vec{q}_{t}$ is the transverse wave vector on the (111) plane. They align well with 


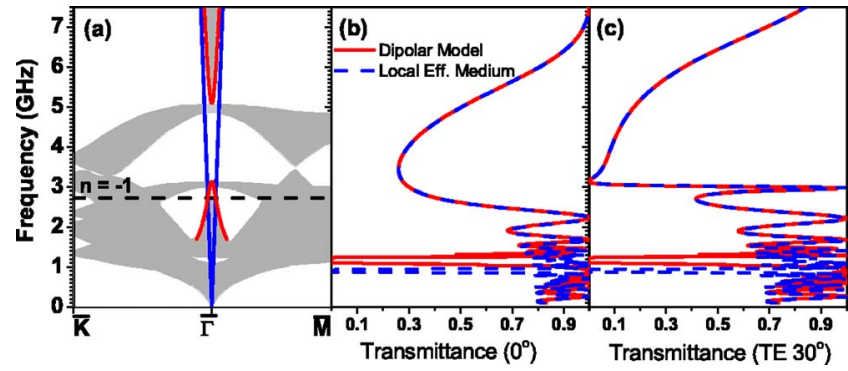

FIG. 3. (Color online) (a) (111)-projected band structure of the full band structure shown in Fig. 2(a). and the TE transmittance on 16 layers of the FCC structure oriented in the [111] direction with (b) normal incidence and (c) oblique incidence of $30^{\circ}$. See text for the detailed description.

the projected band structure. The two blue lines define the light cone outside the slab. It stays very close to the zone center $(\bar{\Gamma})$ as the wavelength $\lambda$ is always much larger than the lattice constant in the whole frequency range. The light cone intersects with the negative refractive band at 2.726 $\mathrm{GHz}(\lambda \approx 110 \mathrm{~mm})$. It is called the focusing frequency, denoted by the horizontal dashed line with a refractive index $n=\sqrt{\varepsilon} \sqrt{\mu}=-1$. At this frequency, all the propagating input waves from a point source in front of the slab can be focused to an image behind the slab so that the source-to-image distance $(l)$ is the double of the slab thickness $(d)$. Here, we only deal with the propagating input waves. The discussion of the subwavelength imaging involving evanescent waves will be dealt with in the next section. In Figs. 3(b) and 3(c), the red solid lines show the transmittance for a slab composed of 16 layers $(d \approx 27.7 \mathrm{~mm})$ of the microstructures oriented in the [111] direction. The results are obtained using the multiple-scattering formulation (layer-KKR method up to the dipolar term ${ }^{22}$ ). The blue dashed lines show the transmittance of the slab if it is replaced by a homogeneous one with effective $\varepsilon$ and $\mu$. Figures 3(b) and 3(c) correspond to the case of normal incidence and 30 degrees oblique incidence for the TE polarization, respectively. We see that the effective medium results for slab transmittance align well with the multiple-scattering results in the whole frequency range except those near the resonance gap (effective medium predicts at $0.9 \mathrm{GHz}$ while the multiple-scattering calculation predicts at around $1.2 \mathrm{GHz}$ ). Since the waves are propagating waves of $\vec{q}_{t}$ very near to the $\bar{\Gamma}$ point, the effective medium represents the bulk very well unless the frequency approaches the resonance gap. In such a case, we have a very large $\varepsilon$ or a very large $\mu$ so that $\left|q_{z} a\right| \ll 1\left(q^{2}=q_{0}^{2} \varepsilon \mu=q_{t}^{2}+q_{z}^{2}\right)$ is violated in the effective medium criteria stated in Eq. (5). The effective medium can only predict the existence of the resonance gap, but with incorrect frequency.

There are some subtle differences between the local effective medium and a system with microstructure even for propagating waves, which reflects the difference between a local theory and a nonlocal theory. As the longitudinal bands do not stay flat within the dipolar model, off-normal incident waves are able to excite the longitudinal modes in the frequency regime where the longitudinal bands and the light cone overlap (near $3.1 \mathrm{GHz}$ or near 5.1 GHz). Figure 4(a)
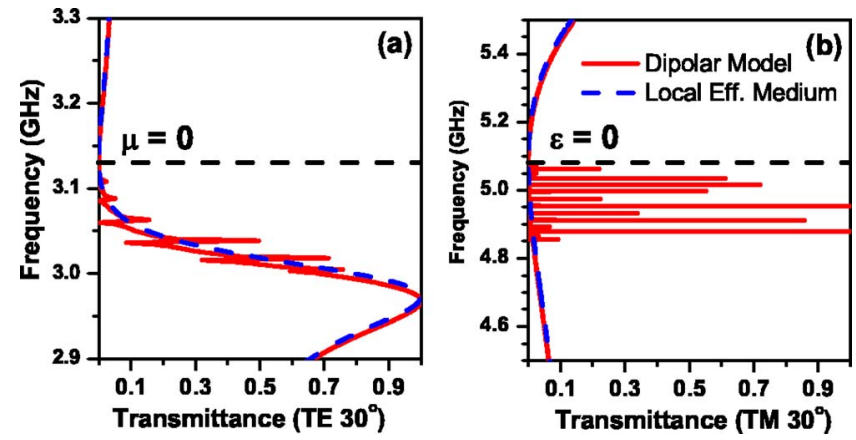

FIG. 4. (Color online) Transmittance for (a) TE wave and (b) TM wave of $30^{\circ}$ oblique incidence on 16 layers of the microstructure in Fig. 2(a) oriented in the [111] direction. The red solid lines show the results by using the dipolar model while the blue dashed lines show the results if the slab is replaced by a homogeneous effective medium.

shows the TE wave transmittance at $30^{\circ}$ oblique incidence near $3.1 \mathrm{GHz}$. It is the magnification of Fig. 3(c). Sharp peaks appear near the frequency of zero permeability. Figure 4(b) shows the corresponding result for TM wave near 5.1 $\mathrm{GHz}$. Sharp peaks appear near the frequency of zero permittivity.

Therefore, there is no essential difference between the dipolar model and its effective medium for the DNG medium near the zone center at low frequencies except near the resonance gap or the longitudinal band. The effective $\varepsilon$ and $\mu$ have useful meaning in describing propagation.

\section{Slab properties, near field}

For an evanescent input wave of $\vec{q}_{t}$ lying outside the light cone but near the $\bar{\Gamma}$ point, we still have small $\left|q_{z} a\right|$ such that the local effective medium should be valid before $q_{t}$ is too large. The validity is crucial for evanescent input waves since it determines how good the image resolution is in slabfocusing as we shall see.

We first examine the surface mode dispersion of the same slab in the preceding section (the modes formed by coupling between the surface modes on the two interfaces). In the local effective medium approximation, the surface mode dispersion is plotted in Fig. 5(a) with blue dashed lines. The upper two bands are for the TM polarization and the lower two bands are for the TE polarization. The surface mode dispersion is obtained from the mode equation

$$
\frac{Z}{Z_{0}}=-\left(\frac{1-e^{i q_{z} d}}{1+e^{i q_{z} d}}\right)^{m},
$$

where $d$ is the thickness of the slab, $Z$ is the surface impedance of the slab defined by

$$
Z^{(\mathrm{TE})}=\frac{\omega \mu}{q_{z}}, \quad Z^{(\mathrm{TM})}=\frac{\omega \varepsilon}{q_{z}},
$$

and $Z_{0}$ is the surface impedance of vacuum defined similarly. The mode number $m$ can either be 1 for an even surface mode of the slab, -1 for an odd surface mode of the slab or 0 for the surface mode of a single interface. The surface 


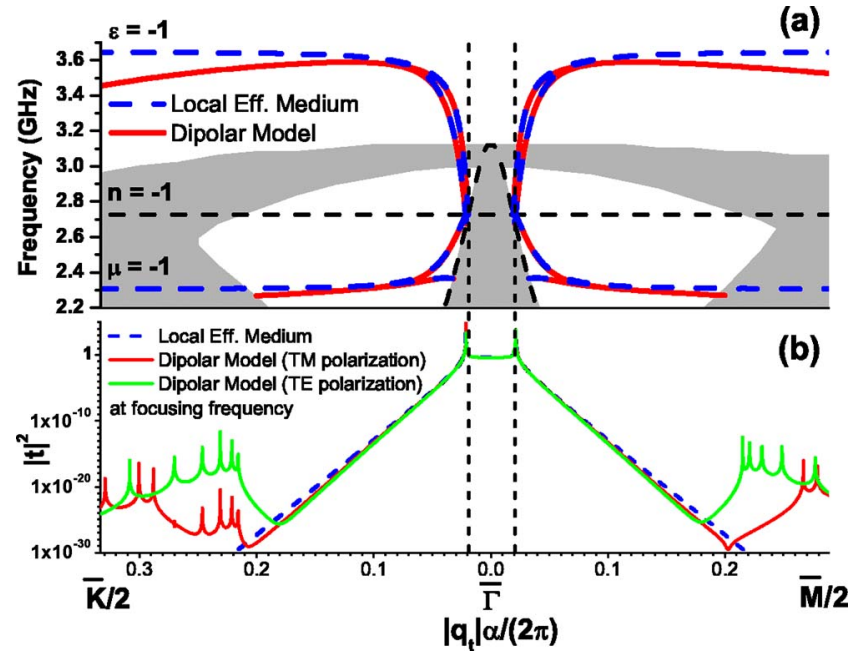

FIG. 5. (Color online) (a) Surface mode dispersion of 16 layers of the microstructure in Fig. 2(a) oriented in the [111] direction. The red solid lines show the result from the dipolar model and the blue dashed lines show the result from the local effective medium description of the same slab. The shaded region shows the projected band structure of the bulk on the (111) plane. The horizontal dashed line denotes the focusing frequency $(n=-1)$. (b) Transfer function from the source to the image at the focusing frequency. See text for the meaning of the symbols.

mode of the slab is characterized as even or odd (in $D_{z}$ field for TM polarization or in $B_{z}$ field for TE polarization) due to the mirror symmetry of the system. If $d$ or $q_{t}$ is large enough, the mode equation can be approximated by

$$
\frac{Z}{Z_{0}} \approx-1+2 m e^{i q_{z} d} .
$$

In this picture, the surface mode splits into an odd and an even surface mode. For $\vec{q}_{t}$ far away from the $\bar{\Gamma}$ point, the surface modes of TE polarization converge to the frequency (2.31 GHz) in which $\mu=-1$ and the surface modes of TM polarization converge to the frequency $(3.65 \mathrm{GHz})$ in which $\varepsilon=-1$.

Figure 6 shows the finer details of the surface mode dispersion near the focusing frequency. At the focusing frequency, the ratio $Z / Z_{0}$ becomes independent of $q_{t}$ [from Eq. (19)] and this ratio for the two polarizations are reciprocal of each other. Then, Eq. (18) can be solved (see the Appendix ) to obtain the position of the surface mode (independent of polarization) by

$$
\left|q_{z}\right|=-\frac{1}{d} \ln \left(\left|\frac{\eta-1}{\eta+1}\right|\right),
$$

where $\eta=\sqrt{\mu} / \sqrt{\varepsilon}$ is the intrinsic impedance of the DNG medium. In this example, $\varepsilon=-2.778, \mu=-0.36$, and hence $\eta$ $=0.36$ is not matched to vacuum (value of one). This is the reason why the surface mode is very near to the light cone unless a thinner slab $(d / \lambda \approx 0.25$ for the current slab) is used. The two surface modes of TE and TM polarizations cross exactly at the focusing frequency with $q_{t} \alpha /(2 \pi)=0.0214[\alpha$

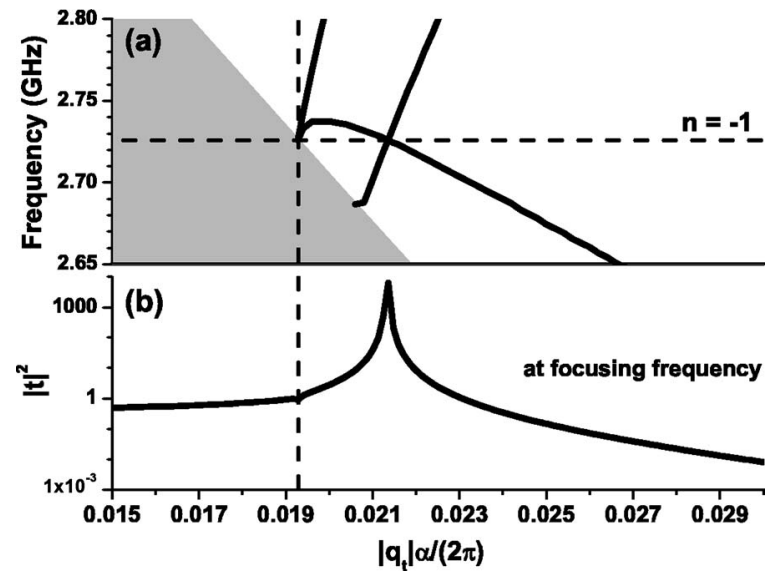

FIG. 6. (a) Surface mode dispersion of 16 layers of the microstructure in Fig. 2(a) oriented in the [111] direction. (b) Transfer function from the source to the image at the focusing frequency. It is the magnification of Fig. 5 near the focusing frequency.

is the inter-resonator spacing for the FCC (111) plane] as shown in Fig. 6(a).

In fact, the position of the surface mode is closely related to the transfer function from the source to the image, denoted by $t$. Figure 5(b) (in blue dashed line) and Fig. 6(b) show the transfer function $t$ at the focusing frequency against $q_{t}$ on both the $\bar{\Gamma} \overline{\mathrm{K}}$ and $\bar{\Gamma} \overline{\mathrm{M}}$ directions in the surface Brillouin zone. The transfer function is degenerate for the two polarizations as expected $\left(Z / Z_{0}\right.$ for the two polarizations are reciprocal of each other). For $q_{t} \alpha /(2 \pi)$ smaller than 0.0193 (shown by the vertical dashed line), we have propagating input waves and the transfer function is in the order of one (but less than 1 since the impedance is not matched). For $q_{t} \alpha /(2 \pi)$ larger than 0.0193, the incident wave is evanescent and the transfer function increases rapidly to a peak indicating the surface mode and then drops exponentially beyond the peak. The image resolution can thus be enhanced beyond the classical diffraction limit. The upper limit of the image resolution is approximately dictated by the $q_{t}$ of the surface mode and the information of the input signal is lost for $q_{t}$ beyond the surface mode. ${ }^{10}$ Here, the resolution enhancement is defined as the ratio between the $q_{t}$ of the surface mode and $q_{0}$ as in Ref. 10. A value of one denotes the classical diffraction limit. In this example, the resolution enhancement is about $0.0214 / 0.0193=1.11$.

Now, we consider the effect of the microstructure on the surface mode dispersion and the transfer function of the lens. The dispersion is calculated by finding out the poles of the transfer matrix of the slab in the layer-KKR method up to the dipolar term. In our calculation, we observe the divergence of the transfer amplitude from the input to the output evanescent waves of the same $\vec{q}_{t}$. The results are shown by the red solid lines in Fig. 5(a). The dispersion with microstructure is well approximated by the local effective medium results near the $\bar{\Gamma}$ point, including the whole regime of propagating input waves $\left[q_{t} \alpha /(2 \pi) \leqslant 0.0193\right]$ and also a small range of the evanescent input waves. The difference between the two becomes significant only in the region far way from the zone center. The dispersion with microstructure does not 
approach asymptotically to the frequencies where $\mu=-1$ or $\varepsilon=-1$. Indeed, it drops below these limits as $q_{t}$ increases further. This spatial dispersion is due to the presence of the microstructure so that the local effective medium theory breaks down as $q_{t}$ increases. ${ }^{16,25}$ The transfer function for both polarizations is also calculated using the layer-KKR method. They are shown in green and red color in Fig. 5(b). It further confirms that the local effective medium is very good near the zone center.

There are extra peaks in the transfer function (with microstructure) as $q_{t}$ further increases when it hits the region of bulk states again (these bulk states cannot be predicted by the local effective medium). These are the traditional waveguide states (standing wave within the slab) and they are negligible due to the small strength in the transfer function. Hence, the local effective medium is good enough when the surface mode is near to the light cone ( $\eta$ not near 1 ).

Suppose we have a thicker and thicker lens (with $\lambda$ and $a$ kept constant) so that the source-to-image distance, $l=2 d$, becomes much greater than $\lambda$. In this limit, all the near fields from the source are expected to decay to negligible values at the image if there are no mechanisms to amplify the near fields. In conventional optics assuming $l \gg \lambda$, we only include the contribution of the far fields but neglect the contribution of the near fields from the source in considering the optical properties of the system. Now, for the DNG slab $(n=1, \eta \neq 1)$ we are considering, near-field amplification exists. The resolution is dictated by the surface mode governed by Eq. (21) which implies that the surface mode shifts to a smaller $q_{t}$ for thicker slab and eventually approaches the light cone as $d \rightarrow \infty$. Therefore, the classical diffraction limit is recovered when $l \gg \lambda$. It agrees with the degradation of resolution displayed by a thicker slab in Ref. 10 and also in Ref. 26 which considers absorption (thicker slab means larger absorption) rather than impedance mismatch of the DNG slab. For example, if we want to obtain the field profile at the image plane for the impedance-mismatched DNG slab when $l \gg \lambda$, only the far fields from the source must be summed in calculating the Green's function of the system.

\section{THE PERFECT LENS WITH MICROSTRUCTURE INCLUDED}

We have just considered the focusing phenomenon of a general situation where $\eta$ of the DNG slab is not matched to vacuum. The local effective medium offers a very good description in such case. Now, we turn to the case $\eta$ approaches one at the focusing frequency. This is the "perfect lens" proposed by Pendry and has drawn considerable attention as well as controversy. The local effective medium of the slab is now changed to the form

$$
\begin{gathered}
\varepsilon(f)=1+\frac{8.62721}{1-f^{2}}, \\
\mu(f)=1+\frac{9}{0.902^{2}-f^{2}},
\end{gathered}
$$

where $f$ is the frequency in GHz. We should call this the impedance-matched configuration. The dipolar resonators are

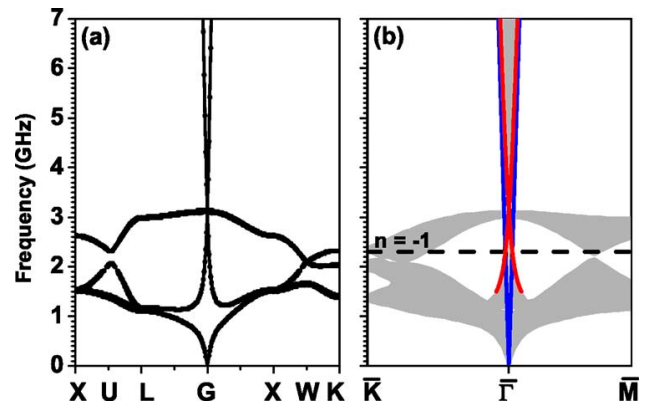

FIG. 7. (Color online) (a) Full band structure and (b) (111)projected band structure extended from the local effective medium given by Eq. (22). The microscopic geometry is the FCC structure with lattice constant of $3 \mathrm{~mm}$.

still placed in an FCC structure with $a=3 \mathrm{~mm}$.

The bulk band structure and the corresponding (111)surface projected band structure are shown in Fig. 7. The light cone and the local effective medium are shown by the blue and red lines as in Fig. 3(a). The local effective medium gives a correct dispersion near the zone center as expected. From Eq. (16), the magnetic and electric bands become degenerate when $\alpha_{e} \approx \alpha_{m}$ which is approximately true for the current configuration. The focusing frequency is at 2.305 $\mathrm{GHz}$ where $n=-1$ while $\eta=1$ up to at least six decimal places from Eq. (22).

When $\eta$ approaches one, it can be proved from Eq. (21) that the position of the surface mode in the local effective medium approximation can be obtained (see the Appendix ) by

$$
q_{t} \approx-\frac{1}{d} \ln \left(\frac{|\delta \eta|}{2}\right),
$$

where $\delta \eta\left(|\delta \eta|<0.5 \times 10^{-6}\right.$ in this example) is the deviation of the intrinsic impedance of the slab from vacuum. From Eq. (23), the surface mode should appear at $q_{t} \alpha /(2 \pi) \geqslant 0.1852$ for a slab of 16 layers of the microstructure oriented in the [111] direction. However, from Fig. 8 which shows the surface modes dispersion and the transfer function at the focusing frequency in red solid lines, the surface mode is at $q_{t} \alpha /(2 \pi)=0.06$, much smaller than the expected value from the local effective medium treatment. It is due to the dispersive behavior of the surface modes at large $q_{t}$ discussed in the preceding section. The dispersive behavior is vital since the nearly perfect lens works by having a very flat surface mode dispersion at the focusing frequency so that the transfer function is kept very near to one along all $q_{t}$ on the surface mode dispersion. Therefore, the flatness of the surface mode dispersion in the local effective medium treatment on the one hand gives perfect or very fine resolution, but on the other hand this mechanism is very sensitive to the system parameters, so that the smallest deviation in frequency, refractive index, impedance [logarithmic dependence in Eq. (23)] or absorption can sharply reduce the resolution. However, when the microstructure is included, which naturally (and inevitably) introduces spatial dispersion, we found that the surface mode dispersion does not stay 


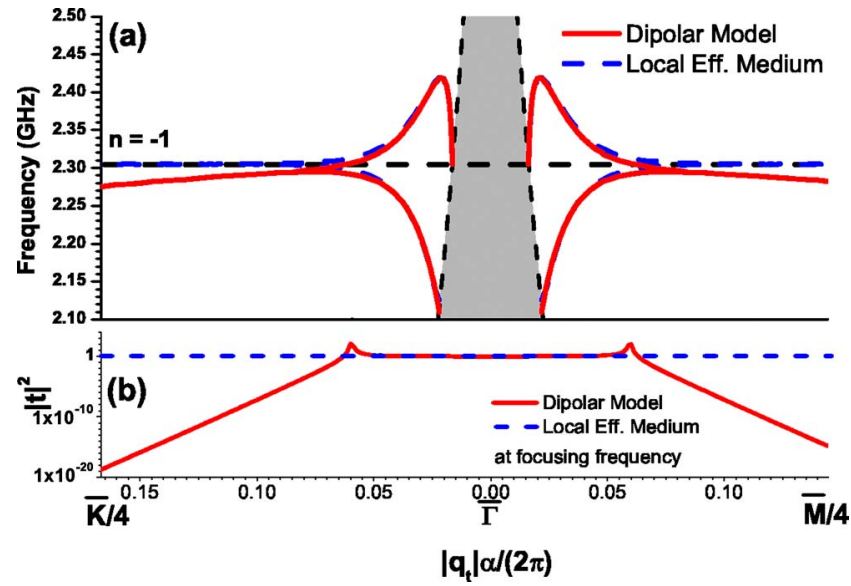

FIG. 8. (Color online) (a) Surface mode dispersion of 16 layers of the microstructure of the impedance-matched configuration [Eq. (22)]. (b) Transfer function from the source to the image at the focusing frequency. The red solid lines show the result from the dipolar model and the blue dashed lines show the result from the local effective medium description of the same slab. The shaded area shows the projected band structure of the bulk on the (111) plane. The horizontal dashed line denotes the focusing frequency at $2.305 \mathrm{GHz}$.

flat. ${ }^{17,18,27}$ The closer to the perfect lens condition, the reduction in the resolution enhancement becomes increasingly dominated by the spatial dispersion due to the underlying microstructure, and other previously discussed effects (such as small deviation in system parameters) become of secondary importance. This is particularly true for metamaterial in which the ratio $\lambda / a$ is usually smaller than a hundred, unlike the case for conventional materials. Moreover, future metamaterials may be fine-tuned to give $\operatorname{Re}(n)=-1$ and $\operatorname{Re}(\eta)=1$ in the local effective medium description exactly ${ }^{20}$ while the absorption is compensated via optical gain. ${ }^{28}$

As the resolution of the perfect lens becomes finite with the existence of the cutoff on $q_{t}$, the field divergence is automatically avoided as not all the near fields emitting from a point source can be amplified. The pole of the perfect lens condition in the parametric space of $\varepsilon$ and $\mu$ is thus regularized. All properties should change smoothly around the perfect lens condition. The transfer function shown in Fig. 8 is qualitatively similar to the case of mismatched impedance shown in Fig. 5 (a rise to a peak at surface mode and then decrease exponentially against $q_{t}$ ). If we change $\varepsilon, \mu$ or $\lambda$ by a small amount, the position of the surface mode and the transfer function should remain nearly the same.

To demonstrate the smoothness around the perfect lens condition, we keep the frequency fixed $\omega \alpha / c=0.1$ ) and vary $n$ or $\eta$ of the DNG slab. In Fig. 9(a), we fix $\eta=1$ and vary $n$, the results for the two polarizations are degenerate no matter the calculation is done with the local effective medium or done with the microstructure since $\varepsilon$ and $\mu$ are the same, so as $\alpha_{e}$ and $\alpha_{m}$. The two polarizations make no difference. The position of the surface mode changes smoothly at around $\left(n, q_{t} \alpha /(2 \pi)\right)=(-1,0.06)$ instead of approaching the horizontal line of $n=-1$. In Fig. 9(b), we fix $n=-1$ and vary $\eta$, the results for the two polarizations are degenerate and ap-

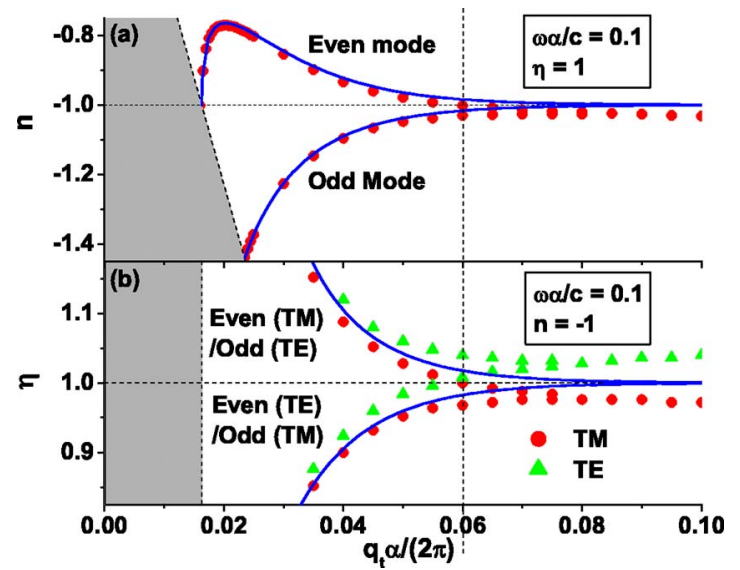

FIG. 9. (Color online) Surface mode dispersion of 16 layers of the microstructure around the perfect lens condition $[(n, \eta)=(-1,1)]$ at a fixed normalized frequency. (a) Results from varying $n$ with $\eta$ fixed at 1 . (b) Results from varying $\eta$ with $n$ fixed at -1 . The dots and triangles show the dispersion from the dipolar model. The solid lines are the corresponding local effective medium prediction. The shaded area denotes the bulk states $\left[q_{t} \alpha /(2 \pi) \leqslant 0.0163\right.$ at the perfect lens condition].

proach $\eta=1$ in the local effective medium treatment. When the underlying microstructure is included, the results for the two polarizations are split and neither result approaches the horizontal line of $\eta=1$ but overshoots and crosses at $\left(\eta, q_{t} \alpha /(2 \pi)\right)=(1,0.06)$ smoothly. Therefore, no matter the lens is working for TE-wave information or TM-wave information, it can only give a maximum resolution enhancement of $0.06 / 0.0163=3.68$.

Finally, we consider the situation when the source-toimage distance $(l)$ becomes much greater than $\lambda$ for the perfect lens. We have already found that only the far fields are important in this limit for a DNG slab with $n=-1$ and $\eta \neq 1$ in the preceding section. The perfect lens in this limit is interesting as in its homogeneous medium version, the perfect lens still works by focusing both the far fields and the near fields. Only a thicker lens $(d=l / 2)$ is needed for a larger $l$. The introduction of the microstructure is expected to impose a maximum cutoff on $q_{t}$. This cutoff is unspecified in the local effective medium theory ${ }^{16}$ and it is expected to be around $\pi / \alpha$, limited by the lattice constant ${ }^{20}$ rather than the wavelength. In this perspective, the resolution enhancement should in principle approach a constant larger than one when $l \gg \lambda$. Figure 10 shows the position of the surface mode against the slab thickness at the focusing frequency for three different cases of lattice constant. The calculations are done with the microstructure. As $d$ increases with both $\lambda(\approx 130 \mathrm{~mm})$ and $a$ kept fixed, the surface mode approaches the light cone and the classical diffraction limit is always recovered when $l \gg \lambda$. For a fixed $d$, we can improve the resolution by reducing the lattice constant of the microstructure. However, special care must be taken in designing the perfect lens with microstructure. For metamaterials in which the resonating units are artificially fabricated, the ratio $\lambda / a$ is usually smaller than 100 . The resolution enhancement falls back to the diffraction limit effectively when the lens is thicker than three wavelengths, as shown in Fig. 10. This 


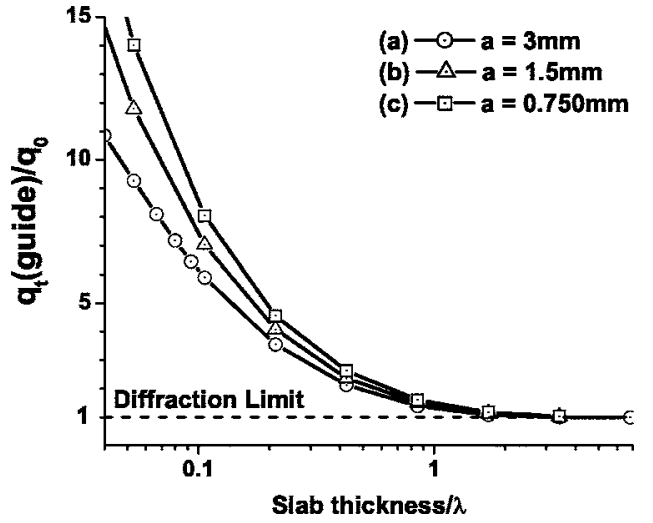

FIG. 10. Position of the surface mode for the slab with microstructure of the impedance-matched configuration against the slab thickness with lattice constants of (a) $3 \mathrm{~mm}$, (b) $1.5 \mathrm{~mm}$, and (c) $0.75 \mathrm{~mm}$ at the focusing frequency. The working wavelength $\lambda$ is at around $130 \mathrm{~mm}$.

result is also true for all the cases at or around the perfect lens condition due to the smoothness demonstrated in Fig. 9.

Therefore, the regularization of the singularity is important here as we want to discuss the physics exactly at the perfect lens condition. The effect of spatial dispersion is incorporated properly by the dipolar model which is universal for any DNG slabs even in approaching the perfect lens condition. Detailed analysis shows that the value $\left|q_{z} d\right|$ of the surface mode for the (nearly) perfect lens is not a constant independent of thickness. This independence is implied from the local effective medium theory [shown by Eq. (21)] or from a perturbative treatment ${ }^{10}$ of the periodicity in the local effective medium. Mathematically speaking, in the local effective medium treatment, the limit $a \rightarrow 0$ is taken before the limit $\eta \rightarrow 1$. It gives rise to the singularity (resolution-field divergence) and a perception that the perfect lens works (by providing a perfect image) when $l \gg \lambda$. In short, no matter whether the impedance is matched or not for the DNG slab at the focusing frequency, it is still safe to include only the far fields in calculating the image properties when the image is far away from the source.

\section{CONCLUSION}

In summary, we have established a microscopic model of DNG media using resonating dipolar building blocks. Comparison of the "exact" result for the dipolar system and the corresponding local effective medium highlights the similarity between the two systems for propagating waves and also some differences for evanescent waves that are important for subwavelength focusing. The model is useful for relating the local effective medium parameters obtained from experiments to nonlocal effective medium theory since in some physical phenomena such as subwavelength focusing, nonlocal feature of the media will become important. Once the microstructure is taken into account, the dispersion of the surface mode appears automatically and inevitably, which imposes resolution limitation to the perfect lens and the field divergence is avoided automatically. In approaching the perfect lens condition, the image properties smoothly change. The resolution enhancement becomes much smaller than the expected value from the local effective medium description. The drop is dominated by the effect imposed by spatial dispersion rather than the impedance mismatch. The super-lens effect disappears when the source-to-image distance is much larger than the working wavelength under the effect of spatial dispersion.

\section{ACKNOWLEDGMENTS}

The authors thank Professor Z. Q. Zhang, Dr. Lei Zhou, and Xinhua $\mathrm{Hu}$ for valuable discussions. This work is supported by RGC Hong Kong through Grant No. CA02/03.SC01.

\section{APPENDIX: SURFACE MODE FOR A SLAB AT THE FOCUSING FREQUENCY}

At the focusing frequency in which the refractive index $n=\sqrt{\mu} \sqrt{\varepsilon}$ is kept at -1 , the surface impedance [defined in Eq. (19)] is given by

$$
\frac{Z^{\mathrm{TE}}}{Z_{0}}=-\eta, \quad \frac{Z^{\mathrm{TM}}}{Z_{0}}=-\frac{1}{\eta},
$$

which is independent of the transverse wave number. In Eq. (A1), $\eta=\sqrt{\mu} / \sqrt{\varepsilon}$ is the intrinsic impedance of the slab. Therefore, by setting Eq. (A1) into Eq. (18), the surface mode equation at the focusing frequency becomes

$$
\left|q_{z}\right|=-\frac{1}{d} \ln \left(\left|\frac{\eta-1}{\eta+1}\right|\right)
$$

which is independent of polarization. When $\eta \approx 1+\delta \eta$ (nearly impedance matched), Eq. (A2) can be further approximated as

$$
q_{t} \approx-\frac{1}{d} \ln \left(\frac{|\delta \eta|}{2}\right) .
$$

It has a similar form to the equation derived in Ref. 10 under the approximation $\varepsilon=-1$ with $\mu=-1+\delta \mu$.
${ }^{1}$ V. G. Veselago, Sov. Phys. Usp. 10, 509 (1968).

${ }^{2}$ R. A. Shelby, D. R. Smith, and S. Schultz, Science 292, 77 (2001).

${ }^{3}$ J. B. Pendry, Phys. Rev. Lett. 85, 3966 (2000).
${ }^{4}$ N. Garcia and M. Nieto-Vesperinas, Phys. Rev. Lett. 88, 207403 (2002).

${ }^{5}$ G. W. 't Hooft, Phys. Rev. Lett. 87, 249701 (2001).

${ }^{6}$ S. A. Ramakrishna, J. B. Pendry, D. R. Smith, D. Schurig, and S. 
Schultz, J. Mod. Opt. 49, 1747 (2002).

${ }^{7}$ R. W. Ziolkowski and E. Heyman, Phys. Rev. E 64, 056625 (2001).

${ }^{8}$ J. B. Pendry, Phys. Rev. Lett. 91, 099701 (2003).

${ }^{9}$ L. Hu and Z. Lin, Phys. Lett. A 313, 316 (2003).

${ }^{10}$ D. R. Smith, D. Schurig, M. Rosenbluth, S. Schultz, S. A. Ramakrishna, and J. B. Pendry, Appl. Phys. Lett. 82, 1506 (2003).

${ }^{11}$ Z. Ye, Phys. Rev. B 67, 193106 (2003).

${ }^{12}$ X. S. Rao and C. K. Ong, Phys. Rev. E 68, 067601 (2003).

${ }^{13}$ R. Merlin, Appl. Phys. Lett. 84, 1290 (2004).

${ }^{14}$ M Nieto-Vesperinas, J. Opt. Soc. Am. A 21, 491 (2004).

${ }^{15}$ G. Gómez-Santos, Phys. Rev. Lett. 90, 077401 (2003).

${ }^{16}$ F. D. M. Haldane, cond-mat/0206420 (unpublished).

${ }^{17}$ R. Ruppin, J. Phys.: Condens. Matter 17, 1803 (2005).

${ }^{18}$ I. A. Larkin and M. I. Stockman, Nano Lett. 5, 339 (2005).

${ }^{19}$ T. Koschny, P. Markos, E. N. Economou, D. R. Smith, D. C. Vier, and C. M. Soukoulis, Phys. Rev. B 71, 245105 (2005).

${ }^{20}$ A. Grbic and G. V. Eleftheriades, Phys. Rev. Lett. 92, 117403
(2004).

${ }^{21}$ J. D. Jackson, Classical Electrodynamics (Wiley, New York, 1999).

${ }^{22}$ N. Stefanou, V. Yannopapas, and A. Modinos, Comput. Phys. Commun. 113, 49 (1998).

${ }^{23}$ D. R. Smith, S. Schultz, P. Markos, and C. M. Soukoulis, Phys. Rev. B 65, 195104 (2002).

${ }^{24}$ V. Yannopapas, A. Modinos, and N. Stefanou, Phys. Rev. B 60, 5359 (1999).

${ }^{25}$ V. M. Agranovich and V. L. Ginzburg, Crystal Optics with Spatial Dispersion, and Excitons (Springer, New York, 1984).

${ }^{26}$ V. A. Podolskiy and E. E. Narimanov, Opt. Lett. 30, 75 (2005).

${ }^{27}$ P. A. Belov, R. Marques, S. I. Maslovski, I. S. Nefedov, M. Silveirinha, C. R. Simovski, and S. A. Tretyakov, Phys. Rev. B 67, 113103 (2003).

${ }^{28}$ S. A. Ramakrishna and J. B. Pendry, Phys. Rev. B 67, 201101(R) (2003). 